Paula de Souza Benevides

\title{
TESTES COMPUTADORIZADOS QUE AVALIAM A ATENÇÃO EM CRIANÇAS
}

\begin{abstract}
Monografia de conclusão de curso apresentada ao Programa de PósGraduação em Psicologia da PUC-Rio como requisito parcial para obtenção do título de especialista em Avaliação

Neuropsicológica.
\end{abstract}

Orientadora: Profa. Dra. Helenice Charchat-Fichman

Rio de Janeiro

Setembro de 2018 
Paula de Souza Benevides

\section{TESTES COMPUTADORIZADOS QUE AVALIAM A ATENÇÃO EM CRIANÇAS}

Orientadora: Profa. Dra. Helenice Charchat-Fichman

Rio de Janeiro

Setembro de 2018 


\section{Sumário}

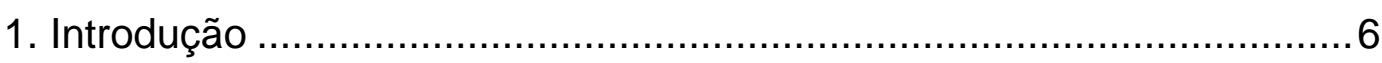

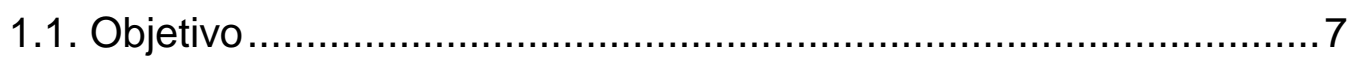

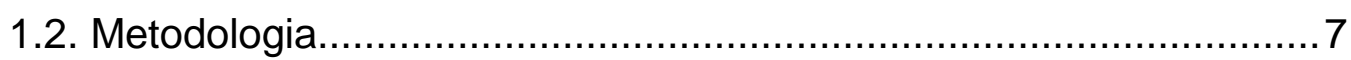

2. Testes tradicionais e computadorizados ....................................... 8

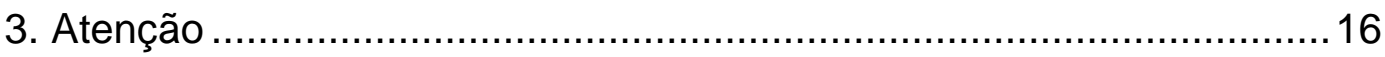

4. Principais testes computadorizados, para criança, que avaliam a

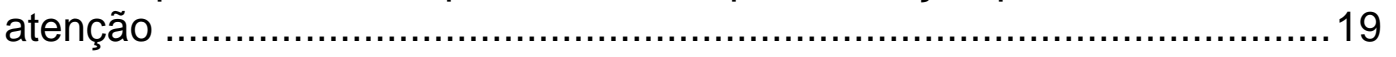

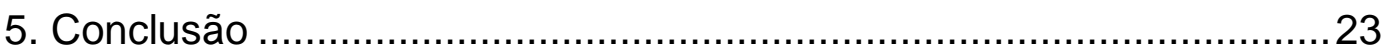

6. Referências Bibliográficas .........................................................23 


\section{Lista de ilustrações}

Tabela 1: Breve descrição de baterias e testes psicológicos e neuropsicológicos, favoráveis para aplicação no Brasil, em crianças. (Fonte: SATEPSI, 2018).

Tabela 2: Descrição de baterias e testes computadorizados (Charchat-Fichman, Uehara e Santos, 2014).

Tabela 3: Baterias e testes computadorizados que avaliam a atenção em crianças (Charchat-Fichman, Uehara e Santos, 2014; SATEPSI, 2018). 


\section{Resumo}

Os testes neuropsicológicos computadorizados apresentam vantagens em relação aos métodos tradicionais. Entretanto, existem diversos fatores que contribuem para a manutenção do uso de baterias baseados em lápis e papel, como a falta de estudos e validações abrangentes. As baterias computadorizadas são capazes de fornecer dados mais precisos e mais rápidos, tornando-se uma grande facilitadora em processos diagnósticos e em reabilitações. Os testes computadorizados validados para crianças ainda são poucos e além disso, carecem de estudos abrangentes de validação. As baterias de testes encontradas que tem como público-alvo as crianças, geralmente têm ênfase em tarefas atencionais.

Palavras-chave: Neuropsicologia, Testes computadorizados, crianças, atenção. 


\section{Introdução}

A neuropsicologia é um campo onde a utilização de testes é algo necessário e recorrente. Para que um instrumento seja elaborado de uma forma ideal, é necessário considerar todo o contexto no qual se pretende aplicar. As diferenças individuais entre a população que terá como alvo, incluindo questões culturais, educacionais, nível socioeconômico e idade são fatores imprescindíveis (P. Schatz \& Browndyke, 2002).

Contudo, apesar do constante uso na área, estudos apontam que os psicólogos utilizam testes, que em sua maioria, foram construídos há muitos anos. Nota-se com isso, a falta de inovação que a Neuropsicologia enfrenta ainda hoje (Rabin, Paolillo, e Barr, 2016).

Existem instrumentos que utilizam lápis e papel, e outros que são os computadorizados. O primeiro deles, mais tradicional, permanece sendo utilizado em grande escala. Mesmo apresentando vantagens superiores às baterias tradicionais, os testes computadorizados enfrentam dificuldades para que sua utilização seja abrangente. As poucas validações encontradas e os obstáculos frente à utilização de novas tecnologias são alguns dos exemplos a serem abordados (Cernich, Brennana, Barker, Bleiberg, 2007).

Os testes computadorizados para crianças possuem menos estudos com ampla validação do que os que têm como público alvo os adultos (Rohlman et al., 2008). Em sua maioria, são instrumentos que pretendem aferir a capacidade atencional das crianças, com o intuito de verificar tanto o desempenho contínuo quanto o tempo de resposta ao estímulo (Charchat-Fichman, Uehara, Santos, 2014).

O presente estudo é composto por três capítulos. O primeiro deles, apresenta os testes tradicionais e os computadorizados, evidenciando suas principais vantagens e desvantagens. Neste capítulo, buscou-se ainda identificar os instrumentos infantis que são favoráveis para aplicação no Brasil, descrevendo a função avaliada por cada um deles Explicitou-se também, os principais testes computadorizados infantis 
encontrados. Já o segundo capítulo aborda a função cognitiva atencional, suas características e subtipos. O terceiro capítulo discorre sobre os testes computadorizados infantis que avaliam a atenção, exemplifica os instrumentos que possuem tais requisitos, além de destacar se possui parecer favorável para utilização no Brasil.

\section{1}

\section{Objetivo}

Apresentar características dos testes tradicionais e computadorizados, com enfoque nos instrumentos infantis computadorizados que avaliem a atenção.

\section{2}

\section{Metodologia}

Trabalho realizado através de revisão de literatura, dividido em três capítulos de fundamentação teórica. O primeiro deles, apresenta os testes tradicionais e os computadorizados, evidenciando suas principais vantagens e desvantagens. Neste capítulo, buscou-se ainda identificar os instrumentos infantis que são favoráveis para aplicação no Brasil, descrevendo a função avaliada por cada um deles Explicitou-se também, os principais testes computadorizados infantis encontrados. Já o segundo capítulo aborda a função cognitiva atencional, suas características e subtipos. O terceiro capítulo discorre sobre os testes computadorizados infantis que avaliam a atenção, exemplifica os instrumentos que possuem tais requisitos, além de destacar se possui parecer favorável para utilização no Brasil. 


\section{Testes tradicionais e computadorizados}

A neuropsicologia clínica vem sofrendo mudanças ao longo do tempo. Por volta das décadas de 1960 e 1970 os clínicos deixaram de utilizar normas que se limitavam ao uso de medidas individuais do funcionamento cerebral para aderirem ao uso de testes validados com bases psicométricas e normativas (Bilder, 2011).

Historicamente, a Neuropsicologia cognitiva tem influência da ciência cognitiva e da teoria de processamento da informação, e cada vez mais, vem se dedicando também ao estudo de redes neurais. Esses modelos computacionais se destacaram no início da neuropsicologia e contribuíram para muitas de suas descobertas científicas (Soto-Pérez, Martín, Angel, Jiménez Gómez, 2010).

Segundo Kane e Kay (1992), na década de 80, acreditava-se que com o avanço da tecnologia haveriam também mudanças nos testes neuropsicológicos, devido ao fato dos computadores começaram a ser vendidos por um valor mais baixo. Naquela época, muitos acreditavam que o computador poderia proporcionar maior controle na administração, na coleta de dados e na pontuação dos testes. Entretanto, os possíveis desafios tecnológicos, a falta de familiaridade com a tecnologia e a possibilidade dos examinadores perderem espaço para as tecnologias acabaram sobressaindo às vantagens oferecidas pela tecnologia (Cernich, Brennana, Barker, Bleiberg, 2007).

Tanto na avaliação neuropsicológica quanto na reabilitação, lápis e papel ainda são muito utilizados, apesar da crescente criação de protocolos tecnológicos. Esses novos protocolos utilizam tecnologia como os tablets, computadores, internet, smartphones e até mesmo videogames (Gonzalez et al., 2003; Horesh, 2001).

Embora existam alguns instrumentos tecnológicos no campo da neuropsicologia, a integração entre os campos da neuropsicologia e da tecnologia ainda não aconteceu por completo, pelo contrário, o uso de tais instrumentos permanece muito restrito (Rabin et al., 2014). Isso faz com que permaneça o uso de métodos desatualizados, que despendem muito tempo tanto para a coleta quanto para a correção dos dados obtidos e fornecem informações aquém do que poderia em relação ao 
comportamento humano. Além disso, são extremamente caros (Collins \& Riley, 2016; Leurent \& Ehlers, 2015).

Para Miller e Barr (2017), as editoras de teste possuem grande interesse em obter fins lucrativos através da venda de manuais, folhas de resposta, livros de estímulo, testes e formulários. Com isso, no cenário, encontram-se de um lado as editoras que não se empenham em fazer uma alteração dos seus produtos para acompanhar os desenvolvimentos tecnológicos e científicos, e de outro, os profissionais que ficam à mercê deste comércio para conseguirem trabalhar.

A falta de inovação nos testes oferecidos para uso no computador contribui ainda mais para a administração dos testes continuarem a ser através de lápis e papel. Ao analisar a validade concorrente entre as medidas computacionais e as analógicas, nota-se que faltam estudos psicométricos necessários, pois, tanto a qualidade quanto a quantidade dos dados normativos computacionais são muito inferiores aos tradicionais (Miller e Barr, 2017).

Segundo Fratti, Bowden e Cook (2017) ; Resch, McCrea e Cullum (2013) houve também falta de investimento na qualidade dos dados normativos e nas propriedades psicométricas, pois, as empresas que passaram a ter controle das vendas de baterias de testes computadorizadas preferiram investir mais na tecnologia em si do produto e menos em propriedades psicométricas. Estudos posteriores demonstraram que muitos desses testes carecem de tais propriedades para uso clínico.

Pesquisas demonstram que ao longo de toda história, os neuropsicólogos vêm utilizando, em sua maioria, testes que tem como padrão o uso de lápis de papel. Uma delas, revelou ainda, que mesmo após 10 anos, os profissionais continuavam aplicando os mesmos testes, provavelmente pela falta de um instrumento computacional que fosse compatível com o tradicional (Rabin, Paolillo, e Barr, 2016).

A pesquisa realizada por Rabin et al., (2016) apontou que num total de 693 testes listados pelos participantes, somente $6 \%$ eram baterias de testes computadorizadas. Outros dados revelaram que quase metade dos profissionais participantes da pesquisa alegaram nunca terem utilizado testes de computador, e apenas $18 \%$ deles disseram que frequentemente faziam uso de metodologia computadorizada. Como possíveis causas para isso, destacam-se: os custos dos testes, a falta de dados normativos adequados e insegurança sobre a utilidade e a validade do teste. 
Surpreendentemente, descobriram que muitos dos testes analógicos utilizados atualmente possuem conceitos teóricos e estímulos desenvolvidos há mais de 100 anos (Rabin et al., 2014).

São muitas as vantagens da utilização de baterias computadorizadas. A precisão que a tecnologia proporciona é, certamente, um dos seus grandes benefícios. Seja ela no controle do tempo, na ordem de apresentação dos estímulos, no registro do tipo de resposta e das latências, e até no desenvolvimento de formas variadas de um mesmo teste (Charchat, Nitrini, Caramelli e Sameshim, 2001). Essa precisão é praticamente impossível de ser alcançada usando testes que necessitam papel e lápis, mesmo quando se utiliza um cronômetro (Kay, Starbuck, 1997; Drasgow, 1993).

Outra importante vantagem é que as baterias computadorizadas não apresentam apenas uma versão de aplicação como as analógicas. A repetida aplicação de um teste no mesmo paciente torna difícil a mensuração da evolução clínica das funções cognitivas devido ao efeito da aprendizagem ocasionado quando há pequenos intervalos de aplicação dos testes (Meulen et al, 2004; Drasgow, 1993).

Além disso, os testes computadorizados garantem maior confiabilidade psicométrica, já que padronizam os estímulos e a coleta dos dados, diminuindo assim, a interferência subjetiva do examinador (Capovilla, 2006; Conklin et al., 2013; Ritsner et al., 2006; P. Schatz e Browndyke, 2002).

Quando comparados aos testes tradicionais facilitam estudos de larga escala (Hervey, Greenfield e Gualtieri (2012) e acarretam custos financeiros bem reduzidos, pois não são necessários gastos relacionados ao papel, por exemplo (CharchatFichman, Nitrini, Caramelli e Sameshima, 2000);

Entretanto, a ansiedade do paciente frente à tecnologia desconhecida pode ser uma desvantagem encontrada ao utilizar testes computadorizados. Em alguns casos, a falta de familiaridade com o instrumento pode tanto ser um limitador quanto a aderência do sujeito, quanto interferir nos resultados encontrados (Browndyke et al., 2002). Quando o paciente se permite utilizar a tecnologia, é necessário que o examinador esteja atento em como se dá essa interação, se as instruções permanecem preservadas na memória de trabalho ou se existe alguma dificuldade de compreensão (CharchatFichman et al., 2000; Kay \& Starbuck, 1997). 
Meulen et al. (2004) destacam que outras limitações encontradas em testes computadorizados são a dificuldade de análise nas respostas orais, as possíveis falhas de interpretação e no entendimento da instrução dada.

Como dito anteriormente, a quantidade e a qualidade da validade psicométrica dos testes com base em lápis e papel são muito maiores. Isso resulta numa validação pouco abrangente dos testes computadorizados, que em sua maioria acabam sendo mais utilizados para fins de pesquisa. (Butcher, Perry e Atlis, 2000; Meulen et al, 2004).

Apesar de haver algumas limitações em agregar a tecnologia às baterias neuropsicológicas, suas vantagens são claramente mais expressivas. Para Charchat et al. (2001), através desta tecnologia é possível realizar diagnósticos precocemente e acompanhar a evolução clínica de muitos transtornos.

As baterias de testes encontradas que tem como público-alvo as crianças, geralmente têm ênfase em tarefas atencionais como o tempo de reação e o desempenho contínuo. O desempenho contínuo pode ser observado através dos erros de omissão, ou seja, quando um alvo deveria ter sido marcado e não foi. A persistência acontece nos casos de respostas impulsivas, e os erros, propriamente ditos, são quando estímulos não alvos são marcados pelo sujeito (CPT; Bloch et al., 2012).

Atualmente, existem muitos instrumentos eletrônicos que acabam utilizando algumas funções cognitivas, como a atenção, a memória e as funções executivas. Alguns exemplos desses equipamentos podem ser os celulares, gravadores, tablets, computadores de forma geral, despertadores, entre outros. Através deles, o sujeito pode tanto se beneficiar usando-o como uma técnica de compensação, quando há algum prejuízo de uma função, quanto utilizá-lo para uma reabilitação. Resultam desta forma, numa maior qualidade de vida ao ampliar a autonomia do paciente. Podem ser utilizados para auxiliar o sujeito em se organizar de forma mais adequada, como nos casos de quem sofre com déficits executivos, mnêmicos ou atencionais. Através deles, o paciente pode se planejar diariamente, diminuindo as chances de esquecer dos seus compromissos e registrar o que foi feito, por exemplo (Inglis et al., 2004).

Essas ferramentas, quando utilizadas na reabilitação, são capazes de desenvolver estratégias de compensação e simular situações diárias com treinos 
ecológicos para que, com o tempo, o paciente consiga aplicar em sua prática (Rizzo et al., 2000).

Apesar do crescente uso de novas tecnologias no contexto da reabilitação neuropsicológica, como nos videogames especiais, foram realizados poucos estudos empíricos sobre suas eficácias e se, posteriormente, ocorre a generalização das estratégias, que são treinadas ao longo do tratamento, no cotidiano dos pacientes (Rivero et al., 2012).

Além disso, são raros os estudos com testes computadorizados, em que houve uma quantidade ideal de validações, para crianças e idosos. Já com os adultos, esse número é um pouco maior (Rohlman et al., 2008).

É imprescindível a realização de novos estudos para o desenvolvimento e validação de baterias baseadas em computadores e protocolos de reabilitação, empregando novas tecnologias de processamento de informação e comunicação. Para que um teste computadorizado seja construído, é necessário considerar as diferenças individuais entre a população que terá como alvo, incluindo questões culturais, educacionais, nível socioeconômico e idade (P. Schatz \& Browndyke, 2002).

Para Miller e Barr (2017) ,deve se tornar uma prioridade geral no campo da neuropsicologia o desenvolvimento de ferramentas orientadas por tecnologia para a coleta de dados comportamentais, orientado pelas demandas clínicas atuais e prever demandas antecipadas que possam vir a surgir. Destacam ainda que se for realizado de forma correta, a mudança mais significativa para o campo será a evolução das ferramentas que os neuropsicólogos usam para coletar e capturar dados. Ressaltam, entretanto, que os componentes centrais da prática clínica devem permanecer compatíveis ao longo do tempo e que o conhecimento dos profissionais permanecerá sendo necessário para impulsionar o desenvolvimento contínuo de novos métodos de coleta de dados e interpretar os dados no contexto do cérebro humano e das relações comportamentais.

Abaixo, a Tabela 1 apresenta os testes tradicionais e computadorizados, disponibilizados no Brasil, que possuem parecer favorável do SATEPSI. Como critério de seleção dos testes a serem apresentados neste trabalho, estão aqueles que possuem a idade inicial de aplicação entre 0 meses e 12 anos. 


\begin{tabular}{|c|c|c|c|}
\hline INSTRUMENTO & $\begin{array}{c}\text { AUTORES } \\
\end{array}$ & FUNÇÃO AVALIADA & CARACTERÍSTICAS \\
\hline $\begin{array}{lr}\text { Escala de } & \text { Maturidade } \\
\text { Mental Colúmbia Edição } \\
\text { Brasileira } & \text { Revisada } \\
\text { (CMMS 3) } & \end{array}$ & $\begin{array}{l}\text { Bessie B. Burgemeister,Carlos } \\
\text { Guilherme Furtado Maciel } \\
\text { Schlottfeldt, Irving Lorge, Lean- } \\
\text { dro Fernandes Malloy-Diniz, Lu- } \\
\text { cille Hollander Blum }\end{array}$ & $\begin{array}{c}\text { Habilidades/Competência, Inteli- } \\
\text { gência,Processos Neuropsicoló- } \\
\text { gicos }\end{array}$ & $\begin{array}{l}\text { Público Alvo: De } 3 \text { a } 12 \text { anos. } \\
\text { Idade da amostra de normatização: } \\
\text { : De } 3 \text { a } 12 \text { anos. } \\
\text { Aplicação: Individual }\end{array}$ \\
\hline $\begin{array}{l}\text { Instrumento de Avaliação } \\
\text { Neuropsicológica Breve } \\
\text { Infantil (NEUPSILIN-Inf } \\
\text { ) }\end{array}$ & $\begin{array}{l}\text { Camila Cruz-Rodrigues, Claudia } \\
\text { Berlim De Mell, Jerusa Fumagalli } \\
\text { De Salles, Maria Alice De Mattos } \\
\text { Pimenta Parente, Mônica Carolina } \\
\text { De Miranda, Rochele Paz Fon- } \\
\text { seca, Thais Barbosa }\end{array}$ & Processos Neuropsicológicos & $\begin{array}{l}\text { Público Alvo: De } 6 \text { a } 12 \text { anos } \\
\text { Idade da amostra de normatização: } \\
\text { De } 6 \text { até } 12 \text { anos } \\
\text { Aplicação: Individual } \\
\text { Não Informatizado }\end{array}$ \\
\hline $\begin{array}{l}\text { Teste de Aprendizagem } \\
\text { Auditivo-Verbal de Rey } \\
\text { (RAVLT) }\end{array}$ & $\begin{array}{l}\text { André Rey, Jonas Jardim De } \\
\text { Paula, Leandro Fernandes Malloy- } \\
\text { Diniz }\end{array}$ & Processos Neuropsicológicos & $\begin{array}{l}\text { Público Alvo: (sem informações) } \\
\text { Idade da amostra de normatização: } \\
\text { (sem informações) } \\
\text { Aplicação: Individual }\end{array}$ \\
\hline $\begin{array}{l}\text { Teste de Atenção Visual - } \\
\text { TAVIS } 4 \text { (TAVIS 4) }\end{array}$ & $\begin{array}{l}\text { Gabriel Coutinho, Neander Abreu, } \\
\text { Paulo Mattos, Pilar Erthal }\end{array}$ & $\begin{array}{c}\text { Processos Neuropsicológi- } \\
\text { cos } \quad \text { Processos perceptivos/cog- } \\
\text { nitivos }\end{array}$ & $\begin{array}{l}\text { Público Alvo: Crianças de } 6 \text { a } 11 \\
\text { anos e adolescentes de } 12 \text { a } 18 \\
\text { anos } \\
\text { Idade da amostra de normatização: } \\
\text { De } 6 \text { até } 17 \text { anos } \\
\text { Aplicação: Individual } \\
\text { Correção: Informatizado }\end{array}$ \\
\hline $\begin{array}{l}\text { Teste Infantil de Memó- } \\
\text { ria - Forma Reduzida } \\
\text { (TIME-R) }\end{array}$ & $\begin{array}{l}\text { Cintia Perez Duarte, Elizeu Cou- } \\
\text { tinho De Macedo,Tatiana Pon- } \\
\text { trelli Mecca }\end{array}$ & $\begin{array}{l}\text { Processos Neuropsicológicos, } \\
\text { Processos perceptivos/cognitivos }\end{array}$ & $\begin{array}{l}\text { Público Alvo: Crianças pré-escola- } \\
\text { res } \\
\text { Idade da amostra de normatização: } \\
\text { De } 3 \text { até } 3 \text { anos } \\
\text { Aplicação: Individual } \\
\text { Correção: Não Informatizado }\end{array}$ \\
\hline Testes de Inteligência & $\begin{array}{l}\text { Acácia Aparecida Angeli Dos } \\
\text { Santos, Ana Paula Porto Noro- } \\
\text { nha, Fabián Javier Marín Rueda, } \\
\text { Fermino Fernandes Sisto, Neli- } \\
\text { mar Ribeiro De Castro }\end{array}$ & Inteligência & $\begin{array}{l}\text { Público Alvo: De } 6 \text { a } 16 \text { anos } \\
\text { Idade da amostra de normatização: } \\
\text { De } 6 \text { Até } 16 \text { Anos } \\
\text { Aplicação: Individual } \\
\text { Não Informatizado } \\
\text { Correção: Informatizado, Não In- } \\
\text { formatizado }\end{array}$ \\
\hline $\begin{array}{l}\text { Escala de Inteligência } \\
\text { Wechsler Abreviada } \\
\underline{\text { WASI })}\end{array}$ & $\begin{array}{l}\text { Clarissa Marceli Trentini, Denise } \\
\text { Balem Yates, Vanessa Stumpf } \\
\text { Heck }\end{array}$ & Inteligência & $\begin{array}{l}\text { Público Alvo: De } 6 \text { A } 89 \text { Anos } \\
\text { Idade Da Amostra De Normatiza- } \\
\text { ção: De } 6 \text { Até } 89 \text { Anos } \\
\text { Aplicação: Individual } \\
\text { Não Informatizado } \\
\text { Correção: Não Informatizado }\end{array}$ \\
\hline $\begin{array}{l}\text { Escala de Inteligência } \\
\text { Wechsler para Crianças } \\
\underline{\text { WISC IV) }}\end{array}$ & $\begin{array}{l}\text { David Wechsler, Elizabeth Do } \\
\text { Nascimento }\end{array}$ & Inteligência & $\begin{array}{l}\text { Público Alvo: De } 6 \text { A } 16 \text { Anos e } 11 \\
\text { meses. } \\
\text { Idade Da Amostra De Normatiza- } \\
\text { ção: De } 6 \text { A } 16 \text { Anos e } 11 \text { meses. } \\
\text { Aplicação:Individual } \\
\text { Não Informatizado }\end{array}$ \\
\hline Escala Geral (MPR) & $\begin{array}{l}\text { J. C. Raven, Maria Cecília De Vi- } \\
\text { lhena Moraes Silva, Silésia Maria } \\
\text { Veneroso Delphino Tosi }\end{array}$ & Inteligência & $\begin{array}{l}\text { Público Alvo: De } 10 \text { A } 69 \text { Anos } \\
\text { Idade Da Amostra De Normatiza- } \\
\text { ção: De } 10 \text { Até } 69 \text { Anos } \\
\text { Aplicação: Individual, Coletivo, } \\
\text { Não Informatizado } \\
\text { Correção: Não Informatizado }\end{array}$ \\
\hline $\begin{array}{lr}\text { Matrizes Progressivas } \\
\text { Coloridas De Raven-Cpm }\end{array}$ & J C Raven, J H Court, J Raven & Inteligência & $\begin{array}{l}\text { Público Alvo: De } 5 \text { a } 11 \text { Anos. } \\
\text { Idade Da Amostra De Normatiza- } \\
\text { ção: De } 5 \text { a } 11 \text { Anos. } \\
\text { Aplicação: Individual, Coletivo, } \\
\text { Não Informatizado. }\end{array}$ \\
\hline $\begin{array}{l}\text { R-2 Teste Não-Verbal De } \\
\text { Inteligência Para Crian- } \\
\text { ças }\end{array}$ & $\begin{array}{l}\text { Helena Rinaldi Ros,Irai Cristina } \\
\text { Boccato Alves }\end{array}$ & Inteligência & $\begin{array}{l}\text { Público Alvo: De } 5 \text { A } 12 \text { Anos } \\
\text { Idade Da Amostra De Normatiza- } \\
\text { ção: De } 5 \text { Até } 12 \text { Anos } \\
\text { Aplicação:Individual, Não Infor- } \\
\text { matizado } \\
\text { Correção: Não Informatizado }\end{array}$ \\
\hline $\begin{array}{l}\text { Teste De Desenvolvi- } \\
\text { mento Do Raciocínio In- } \\
\text { dutivo (Tdri) }\end{array}$ & $\begin{array}{l}\text { Cristiano Mauro Assis Gomes, } \\
\text { Hudson Fernandes Golin }\end{array}$ & Desenvolvimento,Inteligência & $\begin{array}{l}\text { Idade Da Amostra De Normatiza- } \\
\text { ção: (Sem Informações) } \\
\text { Aplicação: Individual, Coletivo } \\
\text { Correção: (Sem Informações) }\end{array}$ \\
\hline
\end{tabular}




\begin{tabular}{|c|c|c|c|}
\hline $\begin{array}{l}\text { Teste De Inteligência Ge- } \\
\text { ral - Não-Verbal (Tig-Nv) }\end{array}$ & $\begin{array}{l}\text { Silésia Maria Veneroso Delphino } \\
\text { Tosi }\end{array}$ & Inteligência & $\begin{array}{l}\text { Público Alvo: De } 10 \text { A } 79 \text { Anos } \\
\text { Idade Da Amostra De Normatiza- } \\
\text { ção: De } 10 \text { Até } 79 \text { Anos } \\
\text { Aplicação: Individual, Coletivo, } \\
\text { Não Informatizado } \\
\text { Correção: Informatizado, Não In- } \\
\text { formatizado }\end{array}$ \\
\hline $\begin{array}{l}\text { Teste De Inteligência } \\
\text { Não-Verbal (Toni-3) }\end{array}$ & $\begin{array}{l}\text { Acácia Santos, Ana Paula Noro- } \\
\text { nha, Fermino Sisto }\end{array}$ & Inteligência & $\begin{array}{l}\text { Público Alvo: De } 6 \text { A } 10 \text { Anos } \\
\text { Idade Da Amostra De Normatiza- } \\
\text { ção: De } 6 \text { Até } 10 \text { Anos } \\
\text { Aplicação: Individual, Não Infor- } \\
\text { matizado } \\
\text { Correção: Não Informatizado }\end{array}$ \\
\hline $\begin{array}{l}\text { Teste Não-Verbal De In- } \\
\text { teligência - Son-R 21/2- } \\
7[\mathrm{~A}]\end{array}$ & $\begin{array}{l}\text { Camila A. Karino, Girlene R. De } \\
\text { Jesus, Jacob A. Laros, Peter J. Tel- } \\
\text { legen }\end{array}$ & Inteligência & $\begin{array}{l}\text { Público Alvo: De } 2 \text { Anos E Seis } \\
\text { Meses A } 7 \text { Anos E } 11 \text { Meses } \\
\text { Idade Da Amostra De Normatiza- } \\
\text { ção: De } 2 \text { Até } 7 \text { Anos } \\
\text { Aplicação: Individual, Não Infor- } \\
\text { matizado. } \\
\text { Correção: Informatizado, Não In- } \\
\text { formatizado }\end{array}$ \\
\hline $\begin{array}{l}\text { Teste Não-Verbal De Ra- } \\
\text { ciocínio Para Crianças } \\
\text { (Tnvri) }\end{array}$ & Luiz Pasquali & Inteligência & $\begin{array}{l}\text { Público Alvo: De } 5 \text { Anos E } 9 \text { Meses } \\
\text { A } 13 \text { Anos E } 3 \text { Meses } \\
\text { Idade Da Amostra De Normatiza- } \\
\text { ção: De } 5 \text { Até } 14 \text { Anos } \\
\text { Aplicação: Individual, Não Infor- } \\
\text { matizado } \\
\text { Correção: Não Informatizado }\end{array}$ \\
\hline
\end{tabular}

Tabela 1. Breve descrição de baterias e testes psicológicos e neuropsicológicos, favoráveis para aplicação no Brasil, em crianças. (Fonte: SATEPSI, 2018).

Quanto aos principais testes computadorizados, estes estão descritos na Tabela 2, com base no estudo realizado por Charchat-Fichman, Uehara e Santos (2014).

\begin{tabular}{|c|c|c|c|c|}
\hline INSTRUMENTO & AUTORES & \multicolumn{3}{|c|}{ FUNÇÃO AVALIADA } \\
\hline ANAM & Reeves et al., 2007 & \multicolumn{3}{|c|}{$\begin{array}{l}\text { Bateria usada para avaliar a precisão e velocidade de processamento, me- } \\
\text { mória e pensamento. }\end{array}$} \\
\hline BTNC/ COMPCOG & $\begin{array}{l}\text { Charchat, } 1999 \text { e Charchat et al., } \\
2001\end{array}$ & \multicolumn{3}{|c|}{$\begin{array}{l}\text { Seis testes neuropsicológicos foram desenvolvidos para avaliar a memória } \\
\text { primária, a memória de curto prazo, o tempo de resposta e a aprendizagem } \\
\text { implícita. Os testes envolvem testes de tempo de resposta simples, tempo } \\
\text { de resposta de escolha, aprendizado implícito, aprendizado de palavras de } \\
\text { curto prazo, aprendizado de imagem de curto prazo e aprendizado de face } \\
\text { de curto prazo. Uma breve bateria conhecida como CompCog foi projetada } \\
\text { para o diagnóstico precoce do Alzheimer, incluindo o tempo de resposta e } \\
\text { a lembrança de rostos como marcadores cognitivos }\end{array}$} \\
\hline CANTAB & $\begin{array}{l}\text { Fray, Robbins, \& Sahakian, 1996; } \\
\text { Wild et al., } 2008\end{array}$ & \multicolumn{3}{|c|}{$\begin{array}{l}\text { A bateria inclui: memória de trabalho e funções executivas; memória visual } \\
\text { e espacial, atenção e tempo de resposta; memória verbal e processos de } \\
\text { tomada de decisão. Estes são avaliados através de } 13 \text { testes. Foi primeira- } \\
\text { mente projetado para diagnosticar a demência }\end{array}$} \\
\hline CNS Vital Signs & $\begin{array}{l}\text { altieri \& Johnson, 2006; Wild } \\
\text { l., } 2008\end{array}$ & \multicolumn{3}{|c|}{$\begin{array}{l}\text { Este teste inclui sete testes envolvendo: memória, velocidade psicomotora, } \\
\text { tempo de resposta, flexibilidade cognitiva e atenção complexa. }\end{array}$} \\
\hline CNTB & $\begin{array}{l}\text { Cutler et al., 1993; Wild et al., } \\
2008\end{array}$ & \multicolumn{3}{|c|}{$\begin{array}{l}\text { Esta bateria é composta por } 11 \text { subtestes que avaliam a velocidade do mo- } \\
\text { tor, o processamento de informações, a atenção, a memória verbal e espa- } \\
\text { cial, a linguagem e as habilidades espaciais. A Bateria de Teste Neuropsi- } \\
\text { cológico Computadorizada (CNTB) é uma das primeiras tentativas de tes- } \\
\text { tar funções cognitivas em um computador. }\end{array}$} \\
\hline $\begin{array}{l}\text { Continuous Performance } \\
\text { Test }\end{array}$ & Conners, 2002 & $\begin{array}{l}\text { Avalia a atenção e a } \\
\text { impulsividade sus- } \\
\text { tentadas e seletivas. }\end{array}$ & $\begin{array}{l}\text { Exemplo 1: O Teste de } \\
\text { Variáveis de Atenção - } \\
\text { TOVA (Dupuy \& } \\
\text { Greenberg, 1993)) }\end{array}$ & $\begin{array}{l}\text { Exemplo 2: Teste de } \\
\text { Desempenho Contínuo } \\
\text { de Matemática - } \\
\text { MATH-CPT (Lufi e } \\
\text { Fichman, 2012) }\end{array}$ \\
\hline COGDRAS-D & $\begin{array}{l}\text { Simpson et al., 1991, Wild et al., } \\
2008\end{array}$ & \multicolumn{3}{|c|}{$\begin{array}{l}\text { Bateria projetada para avaliar os efeitos positivos e negativos das drogas } \\
\text { sobre a cognição. Ajustado para ser usado com demência. A bateria inclui } \\
\text { oito subtestes. }\end{array}$} \\
\hline CogState & $\begin{array}{l}\text { Hammers et al., 2012; Wild et al, } \\
2008\end{array}$ & \multicolumn{3}{|c|}{$\begin{array}{l}\text { Os subtestes incluem tempo de resposta simples, de escolha e complexo, } \\
\text { monitoramento contínuo, memória de trabalho, relacionamentos, aprendi- } \\
\text { zagem incidental e associativa. }\end{array}$} \\
\hline
\end{tabular}




\begin{tabular}{|c|c|c|}
\hline $\begin{array}{l}\text { Cognitive Stability Index } \\
\text {-CSI }\end{array}$ & $\begin{array}{l}\text { Erlanger et al., 2002; Wild et al., } \\
2008\end{array}$ & $\begin{array}{l}\text { Inclui quatro componentes (memória, atenção, velocidade de resposta e ve- } \\
\text { locidade de processamento) com base em } 10 \text { subtestes. Autores sugerem o } \\
\text { Índice de Estabilidade Cognitiva (CSI) para rastrear e monitorar mudanças }\end{array}$ \\
\hline MCIS & Wild et al., 2008 & Versão baseada em computador da tarefa de lista de palavras CERAD. \\
\hline MicroCog & Powell et al., 1993 & $\begin{array}{l}\text { Uma das primeiras baterias de computador desenvolvidas comercialmente } \\
\text { para detectar sinais precoces de comprometimento cognitivo. Ele avalia } \\
\text { memória, atenção, tempo de resposta, habilidades, pensamento visual e es- } \\
\text { pacial e habilidades de cálculo. }\end{array}$ \\
\hline Mindstreams & $\begin{array}{l}\text { Ritsner et al., 2006; Wild et al., } \\
2008\end{array}$ & $\begin{array}{l}\text { Projetado para detectar MCI. Esta bateria é composta de } 9 \text { subtestes: me- } \\
\text { mória verbal, memória não-verbal, inibição de resposta, interferência de } \\
\text { Stroop, resolução de problemas, imagens visuais e espaciais, rimas verbais, } \\
\text { nomeação, velocidade de processamento e planejamento visual e motor. } \\
\text { Muitos desses testes são adaptações de papel e lápis }\end{array}$ \\
\hline CalCAP & $\begin{array}{l}\text { E. N. Miller, 1996; E. N. Miller, } \\
\text { Satz, \& Visscher, } 1991\end{array}$ & Tempo de resposta por escolha - Dígitos e tempo de resposta sequencial. \\
\hline $\begin{array}{l}\text { Groton Maze Learning } \\
\text { Test -GLMT }\end{array}$ & Pietrzak et al., 2008 & $\begin{array}{l}\text { O Groton Maze Learning Test (GMLT) é uma medida neuropsicológica } \\
\text { recente baseada em computador que avalia a memória primária de curto } \\
\text { prazo e as informações visuais e espaciais. Também é sensível a encontrar } \\
\text { erros, persistência e atraso no processamento de informações em adultos } \\
\text { saudáveis. }\end{array}$ \\
\hline IntegNeuro & Williams et al., 2008 & $\begin{array}{l}\text { Avalia a função motora, a atenção, a aprendizagem e a memória, a fluência, } \\
\text { a função executiva e a inteligência estimada. }\end{array}$ \\
\hline TAVIS-IV & $\begin{array}{l}\text { Duchesne \& Mattos, } 1997 \text { (TA- } \\
\text { VIS III). } \\
\text { Gabriel Coutinho, Neander Abreu, } \\
\text { Paulo Mattos, Pilar Erthal (TAVIS } \\
\text { IV). }\end{array}$ & $\begin{array}{l}\text { Avalia a concentração, habilidades seletivas e atenção seletiva em três ta- } \\
\text { refas. }\end{array}$ \\
\hline
\end{tabular}

Tabela 2. Breve descrição de baterias e testes computadorizados (Charchat-Fichman, Uehara

e Santos, 2014).

Tendo em vista que o presente trabalho tem como foco principal os testes compu-

tadorizados de atenção infantis, a seguir será descrito esta função. 


\section{3}

\section{Atenção}

A atenção é uma função que demanda cuidado ao ser avaliada. Fatores como fome, sono, cansaço e uso de drogas psicoativas podem influenciar seu desempenho. Além disso, vale ressaltar que o nível de atenção varia ao logo do dia. Por esses motivos, é necessário cautela ao analisar o desempenho em algum item, pois um desempenho inferior ao esperado em apenas um item, pode não significar um comprometimento severo desta função (Malloy et al., 2010).

Uma característica importante que atenção tem é a forte dependência de interesse e necessidade que a tarefa proporciona ao sujeito. Tarefas em que o indivíduo apresenta mais interesse, resulta certamente em um maior nível atencional, por exemplo. Alguns autores consideram alguns componentes da atenção como pertencentes às funções executivas (Malloy et al., 2010).

Baseados em alguns estudos, autores definiram a existência de três redes neuronais no circuito atencional. Uma delas possui estreita ligação com o estado de alerta. Malloy et al. (2010) denomina esta rede como rede de vigilância, possui relação com o neurotransmissor noraepinefrina e se localiza no córtex frontal direito e parietal. Segundo Sternberg (2016) esse neurotransmissor mantém o estado de alerta. $\mathrm{O}$ autor defende ainda que uma disfunção desta rede pode estar ligada ao TDAH. Outra rede, denominada por Malloy et al. (2010) como rede de atenção visual, se relaciona à seleção dos estímulos, e envolve estruturas como a junção parietal temporal, o colículo superior, os campos oculares frontais e o lóbulo parietal superior. Nesse caso, o neurotransmissor responsável é a acetilcolina (Sternberg, 2016). A terceira rede, chamada de rede executiva (Malloy et al., 2010), agrega estrutas como os gânglios basais, o córtex cingulado lateral ventral, anterior e também o pré frontal. Nesse caso, o neurotransmissor responsável é a dopamina (Sternberg, 2016). 
A atenção pode ser explicada através de três subtipos: atenção concentrada, alternada e dividida. Quanto função, pode participar na seleção de estímulos, na vigilância e detecção de sinais, e na busca de estímulos (Sternberg, 2016).

Para Malloy et al. (2010), a atenção alternada é aquela que nos permite alternar entre um estímulo e outro, sucessivamente. Já a atenção dividida consiste em focar em diferentes estímulos ao mesmo tempo. Entretanto, existe uma divergência entre opiniões de alguns autores. Alguns defendem que seja possível focar em estímulos distintos simultaneamente, e outros alegam que essa rápida troca de foco não seria uma atenção dividida, mas sim uma atenção alternada. Para Sternberg (2016), a atenção dividida ocorre através de uma distribuição do foco quando mais de uma tarefa acontece ao mesmo tempo. Defende ainda que essa divisão possui estreita relação com os níveis de automatização das tarefas e com os níveis de inteligência.

Apesar do constante uso da atenção dividida, Sternberg (2016) alerta que com a realização de tarefas simultaneamente, é provável ocorrer uma diminuição na velocidade de execução destas e também, um aumento na quantidade de erros cometidos.

Para Malloy et al. (2010), a atenção concentrada, também chamada de sustentada é aquela que permite manter-se focado por um longo tempo. Atrelado à esse conceito, encontra-se o papel que o tempo de reação desempenha sobre esse tipo de atenção. $\mathrm{O}$ autor defende a ideia de que diferentes tempos de reação resultam em uma variedade de desempenho da atenção concentrada.

Por meio da seleção de estímulos, obtém-se a atenção seletiva. Por ela o sujeito é capaz de selecionar estímulos mais relevantes em detrimento de outros. A atenção seletiva torna possível focar em estímulos que consideramos serem mais importantes quando comparamos aos outros (Malloy et al., 2010). Esse foco auxilia a execução de mais de um processo cognitivo simultaneamente (Sternberg, 2016).

Já a detecção de sinais e vigilância, tem relação com a manutenção da atenção em uma situação, e consequentemente, sua ação quando é ativada por estímulos pré-determinados desse contexto. Podem -se destacar a amígdala e o tálamo como as principais estruturas envolvidas nesse processo. A amigdala está relacionada ao reconhecimento de estímulos emocionais (Sternberg, 2016). 
A busca por estímulo seria como um rastreio, ou seja, uma procura por algum objeto específico. Para que isso aconteça, e necessária a participação de estruturas do córtex parietal posterior, campos oculares frontais e córtex dorsolateral pré-frontal (Sternberg, 2016).

A atenção se relaciona com outras funções. Por essa razão, um déficit atencional pode causar comprometimentos em outras funções. Até mesmo as habilidades perceptivas, sejam conscientes ou não (Sternberg, 2016), necessitam da atenção para que sejam realizadas de forma adequada (Malloy et al., 2010). 


\section{4 \\ Principais testes computadorizados para avaliar a aten- ção em crianças}

Ainda hoje, os testes computadorizados validados para crianças são poucos e além disso, carecem de estudos abrangentes de validação (Rohlman et al., 2008). Os que encontramos no mercado, em sua maioria, são testes com ênfase em tarefas de atenção, que verificam tanto o desempenho contínuo quanto o tempo de resposta ao estímulo. Através de testes que avaliam tal função, em geral, é possível analisarmos algumas características quanto às respostas dadas pelo paciente. $\mathrm{O}$ desempenho contínuo é verificado através dos erros de omissão, ou seja, quando um estímulo deveria ter sido marcado e não foi. Já a persistência é identificada muitas vezes pelas respostas impulsivas. E os erros, propriamente ditos, são aqueles onde o indivíduo optou por estímulos que não deveriam ter sido assinalados. (CharchatFichman, Uehara, Santos, 2014).

O CPT (Continuous Performance Test) é um instrumento amplamente utilizado capaz de auxiliar em um processo diagnóstico de TDA e de mensurar aspectos relacionados à atenção, como a sustentação e a seleção (CPT; Bloch et al., 2012; Conners, 2002). No momento, existem algumas versões dele, e a mais comercial é a Conners (CPT II). Esta versão avalia a atenção sustentada através de um teste computadorizado visual, utiliza letras como estímulo, e tem duração aproximada de 14 minutos e pode ser aplicado em crianças a partir dos 6 anos de idade (Epstein et al., 2003; Miranda et al., 2008). Outra versão é o K- CPT (Conner’s Kiddie CPT), que é destinado para a aplicação em crianças pré escolares e sua aplicação leva cerca de 7 minutos e ocorre através de estímulos com figuras. Mahone et al. (2005) destacam a prevalência de testes validados para crianças escolares quando comparados aos pré escolares, por esse motivo, o uso do K-CPT tem sido muito relevante na área.

O INTEGNEURO é um teste computadorizado, desenvolvido por Williams et al., 2008, que avalia a função motora, a atenção, a aprendizagem e a memória, a 
fluência, a função executiva e a inteligência estimada, em indivíduos entre 6 e 96 anos .

Existem casos em que um teste é validado para um tipo de população, ou para um propósito, e posteriormente mostra sua eficiência para outro grupo de referência, como o instrumento TOVA (The Test of Variables of Attention (Bloch et al., 2012). Schatz, Ballantyne e Trauner (2001) destacam que tal instrumento, inicialmente, foi elaborado para averiguar desempenho matemático, porém, demostrou grande utilidade ao ser capaz de auxiliar no diagnóstico de TDAH.

Outro caso em que isso ocorreu foi o instrumento CANTAB (Sahakian, Owen, 1992). Inicialmente proposto para o diagnóstico de demência em idosos, mostrou-se eficiente ao avaliar em crianças, a memória de trabalho, as funções executivas, a memória visual e espacial, a atenção, o tempo de resposta, a memória verbal e os processos de tomada de decisão (Fried et al., 2012; Luciana, 2003; Charchat-Fichman, Uehara e Santos, 2014 ).

O principal teste computadorizado, no Brasil, validado para crianças e adolescentes é o TAVIS III. Ele é capaz de verificar a atenção concentrada, a alternada e a seletiva por meio de três tarefas, mostrando ser eficiente no auxílio de diagnóstico de TDA (Duchesne, Mattos, 1997).

Atualmente, outros estudos têm sido relevantes ao avaliar outras funções além da atenção, como era o mais ocorrido. Brooks e Sherman (2012), demonstraram através de estudos, que o CNS VITAL SIGNS é uma bateria eficiente em casos de epilepsia, por exemplo. O instrumento mostrou-se capaz de avaliar funções como a memória, a flexibilidade cognitiva, a latência e a atenção de forma ampla (Gualtieri \& Johnson, 2006; Wild et al., 2008).

A Tabela 3 apresenta os testes computadorizados que avaliam a atenção em crianças. Vale ressaltar que, no Brasil, apenas o TAVIS IV possui parecer favorável para aplicação segundo às normas do SATEPSI. 


\begin{tabular}{|c|c|c|c|c|}
\hline INSTRUMENTO & AUTORES & \multicolumn{3}{|c|}{ FUNÇÃO AVALIADA } \\
\hline $\begin{array}{l}\text { Continuous Performance } \\
\text { Test }\end{array}$ & Conners, 2002 & $\begin{array}{l}\text { Avalia a atenção e a } \\
\text { impulsividade sus- } \\
\text { tentadas e seletivas. }\end{array}$ & $\begin{array}{l}\text { Exemplo 1: O Teste de } \\
\text { Variáveis de Atenção - } \\
\text { TOVA (Dupuy \& } \\
\text { Greenberg, 1993)) }\end{array}$ & $\begin{array}{l}\text { Exemplo 2: Teste de } \\
\text { Desempenho Contínuo } \\
\text { de Matemática - } \\
\text { MATH-CPT (Lufi e } \\
\text { Fichman, 2012) }\end{array}$ \\
\hline IntegNeuro & Williams et al., 2008 & \multicolumn{3}{|c|}{$\begin{array}{l}\text { Avalia a função motora, a atenção, a aprendizagem e a memória, a fluência, } \\
\text { a função executiva e a inteligência estimada. }\end{array}$} \\
\hline CANTAB & $\begin{array}{l}\text { Fray, Robbins, \& Sahakian, 1996; } \\
\text { Wild et al., } 2008\end{array}$ & \multicolumn{3}{|c|}{$\begin{array}{l}\text { A bateria inclui: memória de trabalho e funções executivas; memória visual } \\
\text { e espacial, atenção e tempo de resposta; memória verbal e processos de } \\
\text { tomada de decisão. Estes são avaliados através de } 13 \text { testes. Foi primeira- } \\
\text { mente projetado para diagnosticar a demência }\end{array}$} \\
\hline TAVIS-IV & Duchesne \& Mattos, 1997 & \multicolumn{3}{|c|}{$\begin{array}{l}\text { Avalia a concentração, habilidades seletivas e atenção seletiva em três ta- } \\
\text { refas. }\end{array}$} \\
\hline
\end{tabular}

Tabela 3. Baterias e testes computadorizados que avaliam a atenção em crianças (Charchat-

Fichman, Uehara e Santos, 2014; SATEPSI, 2018). 


\section{Conclusão}

Diante do que foi abordado acima, alguns dos obstáculos enfrentados são a escassez de validação adequada e de estudos sobre o tema, que ocasionam a falta de aprimoramento evidenciada no campo da Neuropsicologia. Como descrito anteriormente, Schatz \& Browndyke, (2002) defendem a importância de realizar novos estudos para o desenvolvimento e validação de baterias computadorizadas, empregando novas tecnologias de processamento de informação e comunicação, sendo imprescindível considerar as diferenças individuais entre a população que terá como alvo, incluindo questões culturais, educacionais, nível socioeconômico e idade.

Outra importante limitação encontrada foi a dificuldade para aderir a tecnologia na rotina clínica. Como justificativa para tal inutilização destaca-se a falta de habilidade com a tecnologia encontrada por pacientes mais velhos, e até mesmo pelos profissionais. Entretanto, esta pode vir a ser uma das suas principais vantagens quando pensamos em sua aplicabilidade em crianças. Isso se deve ao fato da grande familiaridade que a nova geração apresenta com tais instrumentos.

Há a necessidade de investir em estudos que promovam uma maior validação de baterias, sobretudo as computadorizadas, visto que são incontáveis as melhorias que a tecnologia poderá proporcionar na área, tais como melhor precisão de respostas, padronização de aplicação e correção, assim como menor custo com materiais.

Vale ressaltar ainda, a necessidade de elaboração de constructos que busquem avaliar outras funções cognitivas além da atenção, já que esta é a função mais avaliada nos instrumentos existentes. 


\section{Referências bibliográficas}

BILDER, R. M.. Neuropsychology 3.0: Evidence-based science and practice. Journal of the International Neuropsychological Society, v.17, pp.7-13, 2011. DOI:10. 1017/S1355617710001396

BLOCH, Y.; FIXMAN, M.; MAOZ, H.; BLOCH, A. M.; LEVKOVITZ, Y.; RATZONI, G.; GAL, G.. Can computerized cognitive tests assist in the clinical diagnosis of attention-deficit hyperactivity disorder? The Journal of Neuropsychiatry and Clinical Neurosciences, v.24, n.1, pp.111-114, 2012. DOI:10.1176/appi.neuropsych.11010014

BROOKS, B. L.; SHERMAN, E. M.. Computerized neuropsychological testing to rapidly evaluate cognition in pediatric patients with neurologic disorders. Journal

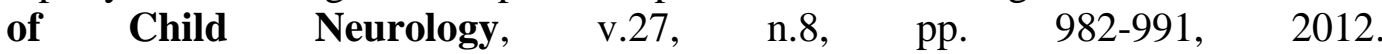
DOI:10.1177/0883073811430863

BROWNDYKE, J. N.; ALBERT, A. L.; MALONE, W.; SCHATZ, P.; PAUL, R. H.; COHEN, R. A.; GOUVIER, W. D. Computer-related anxiety: Examining the impact of technology-specific affect on the performance of a computerized neuropsychological assessment measure. Applied Neuropsychology, v.9, n.4, pp.210$218,2002$.

BUTCHER, J.N.; PERRY, J.N.; ATLIS, M.M.. Validity and utility of computerbased test interpretation. Psychol Assess, v.12, pp.6-18, 2000.

CAPOVILLA, A. G. S.. Desenvolvimento e validação de instrumentos neuropsicológicos para avaliar funções executivas. Avaliação Psicológica, v.5, n.2, pp.239241, 2006.

CHARCHAT-FICHMAN, H.; NITRINI, R.; CARAMELLI, P.; SAMESHIMA, K. (2001). Investigação de marcadores clínicos dos estágios iniciais da doença de Alzheimer com testes neuropsicológicos computadorizados. Psicologia: Reflexão e Crítica, v.14, n.2, pp. 305-316, 2001.

CHARCHAT-FICHMAN, H.; NITRINI, R.; CARAMELLI, P.; SAMESHIMA, K. Contribuição da avaliação neuropsicológica computadorizada no diagnóstico da doença de Alzheimer. In M. de J. Gonçalves, E. C. de Macedo, A. L. Sennyey, \& F. C. Capovilla (Eds.), Tecnologia em (re) habilitação cognitiva. São Paulo, SP: Centro Universitário São Camilo, pp. 110-116, 2000.

CHARCHAT-FICHMAN, H.; UEHARA, E.; SANTOS, C.F.. New Technologies in Assessment and Neuropsychological Rehabilitation. Temas em Psicologia, v.. 22, n.3, pp.539-553, 2014. DOI: 10.9788/TP2014.3-01

CERNICH, A. N.; BRENNANA, D. M.; BARKER, L. M.; BLEIBERG, J.. Sources of error in computerized neuropsychological assessment. Archives of Clinical Neuropsychology, v.22, pp.39-S48, 2007. DOI:10.1016/j.acn.2006.10.004 
COLLINS, F. S.; RILEY, W. T.. NIH's transformative opportunities for the behavioral and social sciences. Science Translational Medicine, v.8, n.366,ed.314, 2016.

CONNERS, C. K.. Conners' continuous performance test. Toronto, Canada: MultiHealth System, 2012.

CONKLIN, H. M.; ASHFORD, J. M.; DI PINTO, M.; VAUGHAN, C. G.; GIOIA, G. A.; MERCHANT, T. E., ...Wu, S Computerized assessment of cognitive late effects among adolescent brain tumor survivors. Journal of Neuro-Oncology, v.113, n.2, p.333- 340, 2013. DOI:10.1007/s11060-013-1123-5

DUCHESNE, M.; MATTOS, P.. Normatização de um teste computadorizado de atenção visual. Arquivos de Neuropsiquiatria, v.55, n.1, pp.62-69, 1997.

EPSTEIN, J. N.; ERKANLI, A.; CONNERS, C. K.; KLARIC, J.; COSTELLO, J. E.; ANGOLD, A. Relations between Continuous Performance Test performance measures and ADHD behaviors. J Abnorm Child Psychol, v.31, n.5, pp.543-54, 2003. Disponível em: < http://psycnet.apa.org/record/2003-07609-006>

FRATTI, S.; BOWDEN, S. C.; COOK, M. J.. Reliability and validity of the CogState computerized battery in patients with seizure disorders and healthy young adults: Comparison with standard neuropsychological tests. The Clinical Neuropsychologist, v.31,pp.569-586, 2017. DOI:10.1080/13854046.2016.1256435

FRIED, R.; HIRSHFELD-BECKER, D.; PETTY, C.; BATCHELDER, H.; BIEDERMAN, J.. How Informative Is the CANTAB to assess executive functioning in children with ADHD? A controlled study. Journal of Attention Disorders, 2012. DOI: $10.1177 / 1087054712457038$

GONZALEZ, R.; GONZALEZ, R.; HEATON, R. K.; MOORE, D. J.; LETENDRE, S.; ELLIS, R. J.; WOLFSON, T.; GRANT, I. Computerized reaction time battery versus a traditional neuropsychological battery: Detecting HIV-related impairments. Journal of the International Neuropsychological Society, v.9, pp.64-71, 2003. DOI:10.1017/S1355617703910071

GUALTIERI, C. T.; JOHNSON, L. G.. Reliability and validity of a computerized neurocognitive test battery, CNS Vital Signs. Archives of Clinical Neuropsychology, v.21, pp.623-643, 2006.

HERVEY, A.S.; GREENFIELD, K.; GUALTIERI, C.T.. Heritability in cognitive performance: Evidence using computer-based testing. Journal of Genetic Psychology, v.173, n.1, p.112-118, 2012. DOI:10. 1080/00221325.2011.573025

HORESH, N.. Self-report vs. computerized measures of impulsivity as a correlate of suicidal behavior. Crisis, v.22, n.1, pp.27-31, 2001. DOI:10.1027//02275910.22.1.27

INGLIS, E.A..; SZYMKOWIAK, A.; GREGOR, P.; NEWELL, A. F.; HINE, N.; WILSON, B. A.; SHAH, P. Usable technology? Challenges in designing a memory aid with current electronic devices. Neuropsychological Rehabilitation, v.14, n.12, p. 77- 87, 2004. DOI:10.1080/09602010343000129

KANE, R. L.; KAY, G. G.. Computerized assessment in neuropsychology: A review of tests and test batteries. Neuropsychology Revie, v.3, n.1, pp.1-117, 1992. 
KAY, G. G.; STARBUCK, V. N.. Computerized neuropsychological assessment. In M. E. Maruish \& J. A. Moses Jr. (Eds.), Clinical neuropsychology: Theoretical foundations for practitioners. Mahwah, NJ: Lawrence Erlbaum Associates, pp. 143-161, 1997.

KERNS, K.A.; RONDEAU, L.A.. Development of a continuous performance test for preschool children. J Atten Disord, v.2, n.4, pp.229-38, 1998.

LEURENT, C.; EHLERS, M. D.. Digital technologies for cognitive assessment to accelerate drug development in Alzheimer's disease. Clinical Pharmacology and Therapeutics, v.98, pp.475-476, 2015. DOI:10.1002/cpt.212.

LUCIANA, M.. Practitioner review: Computerized assessment of neuropsychological function in children: Clinical and research applications of the Cambridge Neuropsychological Testing Automated Battery (CANTAB). Journal of Child Psychology and Psychiatry, v. 44, n.5, pp.649-663, 2003. DOI:10.1111/14697610.00152

COUTINHO, G.; MATTOS, P.; ABREU, N.. Atenção. In: MALLOY-DINIZ (Org). Avaliação Neuropsicológica, pp.86-93. Porto Alegre: Artmed, 2010.

MAHONE, E.M.; PILLION, J.P.; HOFFMAN, J.; HIEMENZ, J.R.; DENCKLA, M.B.. Construct validity of the auditory continuous performance test for preschoolers. Dev Neuropsychol, v.27, n.1, pp.11-33, 2005.

MEAD A.D.; DRASGOW F. Equivalence of computerized and paper-and-pencil cognitive ability tests: a meta-analysis. Psych Bull, v.114, pp.449-458, 1993.

MEULEN E.F.; SCHMAND B.; VAN CAMPEN J.P. The seven minute screen: a neurocognitive screening test highly sensitive to various types of dementia. J Neurol Neurosurg Psychiatry, v.75, pp.700-705, 2004.

MILLER, J.B.; BARR, W.B.. The Technology Crisis in Neuropsychology. Archives of Clinical Neuropsychology. v.32 pp. 541-554. 2017.

MIRANDA, M.C.; SINNES, E.G.; POMPEIA'S; BUENO, O.F.A.. O K-CPT em uma amostra brasileira: descrição do desempenho e comparação com as normas norte-americanas. Rev. psiquiatr. Rio Gd. Sul, vol.31, n.1, pp.60-66, 2009. ISSN 0101-8108. Disponível em: http://dx.doi.org/10.1590/S0101-81082009000100011

RABIN, L. A.; SPADACCINI, A. T.; BRODALE, D. L.,; GRANT, K. S.; ELBULOK-CHARCAPE, M. M.; BARR, W. B... Utilization rates of computerized tests and test batteries among clinical neuropsychologists in the United States and Canada. Professional Psychology: Research and Practice, v.45, pp.368, 2014.

RABIN, L. A.; PAOLILLO, E.,; BARR, W. B.. Stability in test-usage practices of clinical neuropsychologists in the United States and Canada over a 10- year period: A follow-up survey of INS and NAN Members. Archives of Clinical Neuropsychology, 2016. DOI:10.1093/arclin/acw007.

RESCH, J. E.; MCCREA, M. A.; CULLUM, C. M.. Computerized neurocognitive testing in the management of sport-related concussion: An update. Neuropsychology Review, v. 23, pp.335-349, 2013. DOI:10.1007/s11065-013-9242-5 
RIVERO, T.S.; QUERINO, E.H.; STARLING-ALVES, I.. Videogame: Seu impacto na atenção, percepção e funções executivas. Revista Neuropsicologia Latinoamericana, v.4, n.2, pp. 38-47. 2012

RITSNER, M. S.; BLUMENKRANTZ, H.; DUBINSKY, T.; DWOLATZKY, T. The detection of neurocognitive decline in schizophrenia using the Mindstreams. $\begin{array}{lllll}\text { Schizophrenia } & \text { Research. } & \text { v.82, n.1, pp.39-49, } 2006 .\end{array}$ DOI:10.1016/j.schres.2005.10.014

RIZZO, A. A.; BUCKWALTER, J. G.; BOWERLY, T.; VAN DER ZAAG, C.; HUMPHREY, L.; NEUMANN, U.; SISEMORE, D.. The virtual classroom: A virtual reality environment for the assessment and rehabilitation of attention deficits. CyberPsychology \& Behavior, v.3, n.3, p. 483-499, 2000

ROHLMAN, D. S.; VILLANUEVA-UY, E.; RAMOS, E. A. M.; MATEO, P. C.; BIELAWSKI, D. M.; CHIODO, L. M.; OSTREA, E. M. JR. Adaptation of the Behavioral Assessment and Research System (BARS) for evaluating neurobehavioral performance in Filipino children. NeuroToxicology, v. 29, p.143-151, 2008

SAHAKIAN, B. J.; OWEN, A. M.. Computerized assessment in neuropsychiatry using CANTAB: Discussion paper. Journal of the Royal Society of Medicine, v. 85, pp.399-402, 1992.

SCHATZ, A. M.; BALLANTYNE, A. O.; TRAUNER, D. A.. Sensitivity and specificity of a Computerized Test of Attention in the diagnosis of attention deficit/hy$\begin{array}{llll}\text { peractivity disorder. 2001. Assessment, v.8, n.4. } & \text {. }\end{array}$ DOI:10.1177/107319110100800401

SCHATZ, P.; BROWNDYKE, J.. Applications of computer-based neuropsychological assessment. Journal of Head Trauma Rehabilitation, v.17, n.5, p. 395410, 2002.

SOTO-PÉREZ, F.; MARTÍN, F.; ANGEL, M.; JIMÉNEZ GÓMEZ, F.. Tecnologías y neuropsicología: hacia una ciber-neuropsicología. Cuadernos de Neuropsicología, v.4, n.2, pp.112-131, 2010.

STERnBERG, R.J.; STERNBERG, K.. Atenção e Consciência. In: Psicologia Cognitiva. 7 Ed. Cengage Learning, 2015.

WILD, K.; HOWIESON, D.; WEBBE, F.; SEELYE, A.; KAYE, J.. Status of computerized cognitive testing in aging: A systematic review. Alzheimer's \& Dementia, v.4, pp.428-437, 2008. DOI:10.1016/j.jalz.2008.07.003 